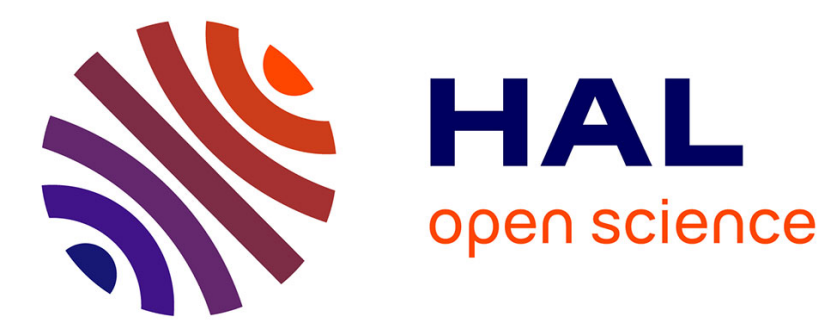

\title{
Giant impacts, heterogeneous mantle heating and a past hemispheric dynamo on Mars
}

Julien Monteux, Hagay Amit, Gael Choblet, Benoit Langlais, Gabriel Tobie

\section{To cite this version:}

Julien Monteux, Hagay Amit, Gael Choblet, Benoit Langlais, Gabriel Tobie. Giant impacts, heterogeneous mantle heating and a past hemispheric dynamo on Mars. Physics of the Earth and Planetary Interiors, 2015, 240, pp.114-124. 10.1016/j.pepi.2014.12.005 . hal-01142028

\section{HAL Id: hal-01142028 \\ https://hal.science/hal-01142028}

Submitted on 16 Jan 2018

HAL is a multi-disciplinary open access archive for the deposit and dissemination of scientific research documents, whether they are published or not. The documents may come from teaching and research institutions in France or abroad, or from public or private research centers.
L'archive ouverte pluridisciplinaire HAL, est destinée au dépôt et à la diffusion de documents scientifiques de niveau recherche, publiés ou non, émanant des établissements d'enseignement et de recherche français ou étrangers, des laboratoires publics ou privés. 


\section{- Giant impacts, heterogeneous mantle heating and a past hemispheric dynamo on Mars}

${ }_{3}$ Julien Monteux $^{a, b, c *}$, Hagay Amit ${ }^{a}$, Gaël Choblet ${ }^{a}$, Benoit Langlais $^{a}$, Gabriel Tobie $^{a}$

$5^{a}$ Laboratoire de Planétologie et Géodynamique, Université de Nantes, CNRS, UMR-6112, 2

6 rue de la Houssinière, 44322 Nantes Cedex, France

$7{ }^{b}$ ISTerre, Université de Grenoble 1, CNRS, Grenoble, France

${ }_{8}{ }^{c}$ Laboratoire Magmas et Volcans, Université Blaise Pascal - CNRS - IRD, OPGC, 5 rue

9 Kessler, 63038 Clermont Ferrand

$10 *$ Corresponding author.

E-mail address: j.monteux@opgc.univ-bpclermont.fr (J. Monteux).

Abstract

The martian surface exhibits a strong dichotomy in elevation, crustal thickness and magnetization between the southern and northern hemispheres. A giant impact has been proposed as an explanation for the formation of the Northern Lowlands on Mars. Such an impact probably led to strong and deep mantle heating which may have had implications on the magnetic evolution of the planet. We model the effects of such an impact on the martian magnetic field by imposing an impact induced thermal heterogeneity, and the subsequent heat flux heterogeneity, on the martian core-mantle boundary (CMB). The CMB heat flux lateral variations as well as the reduction in the mean CMB heat flux are determined by the size and geographic location of the impactor. A polar impactor leads to a north-south hemispheric magnetic dichotomy that is stronger than an east-west dichotomy created by an equatorial impactor. The amplitude of the hemispheric magnetic dichotomy is mostly controlled by the horizontal Rayleigh number $R a_{h}$ which represents the vigor of the convection driven by the lateral variations of the CMB heat flux. We show that, for a given $R a_{h}$, an impact induced CMB heat flux heterogeneity is more efficient 
than a synthetic degree-1 CMB heat flux heterogeneity in generating strong hemispheric magnetic dichotomies. Large $R a_{h}$ values are needed to get a dichotomy as strong as the observed one, favoring a reversing paleo-dynamo for Mars. Our results imply that an impactor radius of $\sim 1000 \mathrm{~km}$ could have recorded the magnetic dichotomy observed in the martian crustal field only if very rapid post-impact magma cooling took place.

Keywords: Mars, dynamo, magnetic field, core-mantle boundary, impact, heat flux.

\section{Introduction}

Giant impacts have strongly influenced the internal structure and dynamics of the terrestrial planets during the primordial stages of their evolutions (Hartmann and Davis, 1975; Benz et al., 1988; Asphaug et al., 2006; Andrews-Hanna et al., 2008; Marinova et al., 2008; Nimmo et al., 2008; Jutzi and Asphaug, 2011). These events are plausible explanations for remarkable features of the solar system such as the small volume of Mercury's mantle relative to its core (Benz et al., 1988; Gladman and Coffey, 2009), the Earth-Moon system (Canup, 2004) and the topographic martian and lunar hemispheric dichotomies (Marinova et al., 2008; Nimmo et al., 2008; Jutzi and Asphaug, 2011). Giant impacts have also been invoked to explain the initiation or cessation of the dynamos of the terrestrial planets and moons (Roberts et al., 2009; ArkaniHamed and Olson, 2010; Reese and Solomatov, 2010; Monteux et al., 2013; Monteux and Arkani-Hamed, 2014). In these models, the impactors' radii typically range between 100 and $1000 \mathrm{~km}$. These impacts deliver a large amount of heat to the deep mantle, which is likely to strongly affect the efficiency of core cooling and in turn the dynamo activity. Although there is a higher probability that a giant impact will fall on low-latitudes of the planetary surface (Le Feuvre and Wieczorek, 2011), true polar wander events can ultimately place the resulting thermal anomaly at high-latitudes of the CMB. Moreover, large impacts could be responsible for significant resurfacing and reset the magnetization of the pre-impact material (Langlais and Thébault, 2011; Lillis et al., 2013).

On Earth, the influence of lower mantle thermal heterogeneity on core magnetohydrodynamics has been extensively studied using numerical dynamos with imposed non-uniform outer boundary conditions. It has been shown that heterogeneous core-mantle boundary (CMB) heat flux causes a deviation from axisymmetry in the core flow (Aubert et al., 2007), in the 
time-average paleomagnetic field (Olson and Christensen, 2002) and in locations of intense magnetic flux patches on millennial time-scales (Bloxham, 2002; Amit et al., 2010). It may also explain the emergence of intense magnetic flux patches in the equatorial region (Amit and Choblet, 2012) and may even yield field locking (Gubbins et al., 2007; Willis et al., 2007). Heterogeneous $\mathrm{CMB}$ heat flux may also recover the lateral variations in the inner-core boundary seismic properties (Aubert et al., 2008; Amit and Choblet, 2009). Finally, reversal frequency and the trajectory of the paleomagnetic dipole axis during reversals may also be governed by the heterogeneous lower mantle (Glatzmaier et al., 1999; Kutzner and Christensen, 2004; Olson et al., 2010, 2013; Olson and Amit, 2014).

Heterogeneous mantle control has also been proposed to explain some features of planetary magnetic fields. Cao et al. (2014) found that high equatorial CMB heat flux breaks the core flow symmetry and produces north-south asymmetric magnetic fields which may explain the observed field of Mercury (Anderson et al., 2012). Stanley (2010) argued that temperature differences in the surrounding envelope of the convective zone of Saturn axisymmetrize its magnetic field. It has also been proposed that CMB heterogeneity may have controlled the shape of the current Martian magnetic field (Stanley et al., 2008). Mars is characterized by a striking magnetic field dichotomy, which is correlated with the topographic dichotomy. The northern lowlands are mostly devoid of significant magnetic fields. In contrast the southern highlands exhibit large and in some places intense magnetic field anomalies, up to $1500 \mathrm{nT}$ at $90 \mathrm{~km}$ altitude as measured by Mars Global Surveyor (Acuña et al., 1998). This is two orders of magnitude larger than the crustal magnetic field on Earth. In terms of magnetized material, this suggests a thick $(40 \mathrm{~km}$ ) and intensely magnetized (up to $12 \mathrm{~A} / \mathrm{m}$ ) lithosphere to produce the observed magnetic field (Langlais et al., 2004), or any combination of a thinner lithosphere and a more intense magnetization (e.g. Parker, 2003).

The martian magnetic dichotomy can be explained using two end-members scenarios. In the external scenario, the dynamo was equally strong in both hemispheres, and the resulting magnetization was equally strong in both hemispheres. Then the magnetization of the northern hemisphere was removed or erased after the dynamo cessation, e.g., by a giant impact (Nimmo et al., 2008) or volcanic activity (Lillis et al., 2008). Alternatively a significant magnetization was never recorded in the northern hemisphere because surface conditions, lithological or 
alteration processes were different from those in the southern hemisphere (Rochette, 2006;

Quesnel et al., 2009; Chassefière et al., 2013). In the internal scenario, the magnetization is strong only in the southern hemisphere because the dynamo was hemispheric to begin with (Langlais and Amit, 2008; Stanley et al., 2008; Amit et al., 2011).

Such an hemispheric dynamo could have been driven by CMB heat flux heterogeneity possibly caused by a very large-scale mantle convection pattern (Harder and Christensen, 1996; Zhong and Zuber, 2001; Elkins-Tanton et al., 2003, 2005; Ke and Solomatov, 2006; Roberts and Zhong, 2006) or by a giant impact (Roberts et al., 2009). In this study we propose a model for the magnetic field dichotomy in which the dynamo hemisphericity (internal origin) is related to a large impact (external origin) (Stanley et al., 2008; Amit et al., 2011; Dietrich and Wicht, 2013). For that purpose, we model heterogeneous CMB heat flux resulting from giant impact heating and investigate its influence on the core dynamo by imposing it as a static, laterally-varying outer boundary condition on numerical dynamo models. In this approach, the CMB heat flux pattern and amplitude, as well as the reduction in the mean heat flux with respect to a reference pre-impact value, are determined by the impactor size, using a synthetic description of the impact heating zone. In section 2 we describe our method. The results are presented in section 3. Discussion, post-impact time evolution and applicability of our results to Mars are given in section 4. Conclusions and possible planetary applications are highlighted in section 5 .

\section{Method}

\subsection{Impact heating at the CMB}

Large impacts brought to Mars a formidable amount of energy that is a function of the impactor mass and velocity, the latter strongly depending on the impacted planet radius $R$. After a large collision on a Mars-size body, a significant fraction of this energy is deeply buried as heat within the mantle and leads to a local temperature increase $\Delta T_{0}$ below the impact site. The size and the shape of the post-impact thermal anomaly depend on several parameters such as the size of the impactor, the impact velocity and angle, and the structure of the martian 
mantle. Increasing the size of the impactor leads to an increase of the heated volume while increasing the impact angle from 0 (head-on impact) to larger values (oblique impacts) reduces the maximal depth reached by the post-impact thermal anomaly (Pierazzo et al., 1997; Pierazzo and Melosh, 2000). Here for simplicity, we consider that the volume of the thermal anomaly only scales with the size of the impactor and we consider the case of a head-on impact. Hence, the post-impact thermal anomaly in our models is approximately uniform within a spherical volume (termed isobaric core) with radius $R_{i c}$ that is 1 to 1.44 times larger than the radius of the impactor $R_{i m p}$ (Pierazzo et al., 1997; Senshu et al., 2002; Monteux et al., 2013).

On Mars, the impactor size invoked to explain the topographic dichotomy ranges between 320 and $1350 \mathrm{~km}$ (Marinova et al., 2008; Nimmo et al., 2008). This has to be compared to the size of the martian mantle. Based on solar tidal deformations, the martian core radius has been estimated between 1520 and $1840 \mathrm{~km}$ (Yoder et al., 2003). For simplicity, we assume a core radius of $1700 \mathrm{~km}$, which implies a mantle thickness of about $1700 \mathrm{~km}$. Hence, considering that $R_{i c}=1.44 R_{i m p}$, the post-impact spherical thermal anomaly is likely to overlap the CMB for $R_{i m p}>500 \mathrm{~km}$. For an impactor radius of $R_{i m p}=1200 \mathrm{~km}$, the disruption of the impacted planet will only occur when the impact velocity reaches values of $\sim 100 \mathrm{~km} / \mathrm{s}$ which is much larger than the impact velocity $v_{i m p}$ considered here $\left(v_{i m p}=5 \mathrm{~km} / \mathrm{s}\right)$ (Tonks and Melosh, 1992; Reese et al., 2010). In our models, we consider that the impactor radius ranges between 600 and $1000 \mathrm{~km}$ bearing in mind that larger impactors with larger impact angles could have similar thermal consequences at the CMB (Pierazzo et al., 1997; Pierazzo and Melosh, 2000).

As the volume of the isobaric core is governed by the size of the impactor, the magnitude of the temperature increase can be directly related to the impact velocity. Making the conservative hypothesis that the impact velocity is close to the martian escape velocity and that the volume of the isothermal sphere is 3 times larger than the impactor (Senshu et al., 2002; Monteux et al., 2013), the energy balance accounting for heating and melting of both the impactor and impacted material may lead to a uniform spherical temperature increase of $\sim 400 \mathrm{~K}$ in the martian mantle (Monteux et al., 2013). Away from the isothermal sphere, the temperature decreases rapidly with distance $r$ as $\left(R_{i c} / r\right)^{m}$ with $m$ typically ranging between 4 and 5 (Senshu et al., 2002; Monteux et al., 2007). 
Geochemical evidence and crater densities indicate that the martian topographic dichotomy could have formed within the first 50 Myr of Solar System formation and that the martian northern hemisphere has been low and stable for nearly all of Mars' history (Zuber, 2001; Frey et al., 2002; Solomon et al., 2005; Marinova et al., 2008). Hence, the impact-driven temperature increase is superimposed to the pre-impact thermal state of the martian mantle that strongly depends on the short-lived radiogenic heating, the accretion processes and the dissipation of gravitational energy during the core formation (Senshu et al., 2002; Golabek et al., 2009; Šrámek et al., 2010). The uncertainties on the relative importance of these processes as well as the diversity of the processes involved in the core formation lead to a wide range of plausible early thermal states after the full differentiation of Mars. For simplicity, we assume here a 1D radially dependent pre-impact mantle temperature field. The choice of this specific temperature field is not crucial as long as the impact heating of the mantle is predominant. As shown later, the main parameter affecting the dynamo is the amplitude of the heat-flux heterogeneity at the CMB. Before the impact heating, we consider a simplified temperature profile as in Monteux et al. (2013) with a CMB temperature of $T=2000 \mathrm{~K}$ and a convective mantle temperature of $\sim 1600 \mathrm{~K}$ (Roberts and Arkani-Hamed, 2012). It should be noted that in reality, the pre-impact thermal state of the martian mantle was probably much more complicated than the one used in our models, with lateral heterogeneities as well as radial variations (including thermal boundary layers and pressure dependence). For simplicity, we do not consider the changes of mantle properties with depth such as the pressure increase and the corresponding adiabatic heating. Because we consider here that the mantle temperature above the CMB is uniform and equal to $1600 \mathrm{~K}$ while the core temperature is $2000 \mathrm{~K}$, adding more complexity should slightly decrease the amplitude of the heat flux heterogeneity. However, since the early martian thermal state is poorly constrained, our simple model may be considered as a reasonable first step to understand the influence of giant impacts on planetary dynamos.

The superposition of the large impact-driven temperature increase leads to a significant perturbation of the pre-impact homogeneous CMB mean heat flux $q_{0}^{h}$. To obtain the postimpact heat flux at the martian CMB for a given impactor radius, we used an impact heating model similar to the one described in Monteux et al. (2013). A uniform spherical temperature anomaly rapidly decreasing with distance is superimposed on the martian pre-impact temper- 
ature field. The impact heating is followed by a thermal re-adjustment within a characteristic time that is governed by the rheology of the mantle surrounding the impact-induced thermal anomaly. This characteristic time is $\tau_{i m p} \sim 10 \mathrm{Myr}$ (Monteux et al., 2007). The characteristic magnetic diffusion time is $\tau_{\lambda}=r_{o}^{2} / \lambda$ where $r_{o}$ is the core radius and $\lambda$ is the magnetic diffusivity (e.g. Bloxham and Jackson, 1991). For Mars $r_{o}=1700 \mathrm{~km}$ and combined with updated estimates of the electrical conductivity of molten iron in Earth's core conditions of $\lambda \sim 0.5$ $\mathrm{m}^{2} / \mathrm{s}$ (Pozzo et al., 2012), these estimates give $\tau_{\lambda} \sim 180 \mathrm{kyr}$. Since $\tau_{\text {imp }}>>\tau_{\lambda}$, the post impact mantle temperature field may be considered constant in our numerical dynamo models.

The shock wave also leads to a temperature increase within the core of the impacted planet, much stronger in the region directly beneath the impact site. The low-viscosity rotating liquid core cannot sustain lateral variations of temperature and the core overturns, resulting in a stably stratified temperature which increases with radius. In the case of a homogeneous CMB heat flux, the thermal stratification occurs within a few kyr (Arkani-Hamed and Olson, 2010). This stratification kills the possible pre-existing core convection, and hence the core dynamo. Then it can take up to several tens of Myr to remove this stratification by conductive core heat loss (Arkani-Hamed and Olson, 2010). However, a significant fraction of the mantle right above the CMB may have experienced melting which facilitated the core cooling. The time needed to remove the core excess heat by convection is $10^{3}-10^{4} \mathrm{yr}$ when considering a molten layer above the CMB (Monteux et al., 2011). It is therefore likely that after the impact the martian dynamo died during the time needed to generate and remove the post-impact core stratification (i.e. $\sim 10^{3}-10^{4} \mathrm{yr}$ ). Then, the dynamo probably re-started, while the impact-driven lower mantle anomaly was still in place (during $\tau_{i m p} \sim 10 \mathrm{Myr}$ ). For simplicity, we do not consider here the core impact heating and the subsequent rapid thermal readjustment. Our dynamo models are convectively unstable throughout the shell, corresponding to the state of the system after core stratification has been removed and while the CMB heterogeneity was still in place. We discuss this aspect in the conclusion section.

As the post-impact mantle temperature reaches the temperature of the core, the heat flux $q$ is nearly zero where the isobaric core overlaps with the CMB. Away from the isobaric core and along the CMB, the heat flux increases rapidly to its pre-impact mean value $q_{0}^{h}$ (Fig. 1). The reduction in the mean $\mathrm{CMB}$ heat flux due to the impact corresponds to the relative $\mathrm{CMB}$ 
surface that is heated by the impactor and is defined by $q_{0}^{r}$ as

$$
q_{0}^{r}=\frac{q_{0}^{h}-q_{0}}{q_{0}^{h}}
$$

where $q_{0}$ is the post-impact mean heat flux. The amplitude of the heat flux heterogeneity is commonly given by $q^{*}$ (Olson and Christensen, 2002) with

$$
q^{*}=\frac{q_{\max }-q_{\min }}{2 q_{0}}
$$

where $q_{\max }$ and $q_{\min }$ are the maximal and minimal values of the post-impact heat flux respectively. In Fig. 1, we show $q_{0}^{r}$ and $q^{*}$ obtained from our post-impact heating model as a function of the impactor size. As the impactor size increases, the extension of the isobaric core on the CMB increases, i.e. $q_{0}$ decreases and therefore $q_{0}^{r}$ and $q^{*}$ increase. An impactor with a radius smaller than $R_{i m p} \sim 500 \mathrm{~km}$ has a negligible effect on the heated surface of the CMB. For small impactors a small portion of the CMB is heated so $q_{0} \simeq q_{0}^{h}$, and since $q_{\max }=q_{0}^{h}$ and $q_{\text {min }}=0$ always hold, for small impactors $q^{*} \simeq 0.5$. An impactor radius of $R_{i m p} \sim 1000 \mathrm{~km}$ decreases the mean CMB heat flux by $26.5 \%$ and produces an heterogeneity with amplitude $q^{*} \sim 0.67$. In our models, we limit the impactor radius to $1000 \mathrm{~km}$ and consider a head-on impact which may represent cases with larger impactors and smaller impact angles (Pierazzo et al., 1997; Pierazzo and Melosh, 2000).

Next we expand the impact-driven CMB heat flux pattern in terms of spherical harmonic coefficients (Fig. 2). In order to use it as an outer boundary condition for heat flux in our dynamo models, we performed a spherical harmonic expansion truncated at $\ell_{\max }=20$ (see Fig. 2 for $R_{i m p}=800 \mathrm{~km}$ ). First we fit the CMB heat flux with the following analytical expression in terms of the angular distance $\omega$ from the impactor's center:

$$
q_{\omega}=\exp \left[-\frac{1}{n}\left(\frac{\omega}{\omega_{0}}\right)^{n}\right]
$$

The Gauss-like function (3) avoids undesirable Gibbs effects associated with discontinuous gradients on the edge of the imprint of the isobaric core on the CMB. The best fit parameters found for the three impactor radii are $\omega_{0}=15^{\circ}$ and $n=3$ for $R_{i m p}=600 \mathrm{~km}, \omega_{0}=30^{\circ}$ and $n=7$ for $R_{i m p}=800 \mathrm{~km}$ and $\omega_{0}=48^{\circ}$ and $n=7$ for $R_{i m p}=1000 \mathrm{~km}$. 


\subsection{Numerical dynamo models}

We solve the set of self-consistent non-dimensional Boussinesq magnetohydrodynamics equations for dynamo action due to thermal convection of an electrically conducting fluid in a rotating spherical shell (for governing equations and more details see Amit et al., 2011). We use the code MAGIC by Johannes Wicht (Wicht, 2002). We analyze numerical dynamos with rigid insulating boundary conditions. The models differ in the imposed outer boundary heat flux pattern and the amplitude of its variation. A summary of model parameters, outer boundary heat flux patterns, geometries and main results is given in Table 1.

It is likely that Mars has no solid inner core up to the present day (e.g. Schubert and Spohn, 1990; Breuer et al., 2010) and, as a consequence, convection in the early martian dynamo was purely thermal (Amit et al., 2011; Dietrich and Wicht, 2013), driven by secular cooling and perhaps by radioactive heating. This convection mode is highly sensitive to CMB heat flux heterogeneity and may thus break internal dynamo symmetries with relatively moderate heterogeneity amplitudes (Hori et al., 2014). Due to numerical singularity at the center of the planet, we retain in our dynamo models a small inner core with a radius $r_{i} / r_{o}=0.2$ of the outer core radius. We impose zero heat flux on the inner boundary so that the inner core is convectively passive. Overall, results by Aubert et al. (2009) and Hori et al. (2010) suggest that such a relatively small and passive inner core has little effect on the dynamo models.

Four internal non-dimensional parameters control the dynamo action. The heat flux Rayleigh number (Olson and Christensen, 2002) represents the strength of buoyancy force driving the convection relative to retarding forces

$$
R a=\frac{\alpha g_{0} q_{0} D^{4}}{k \kappa \nu}
$$

where $\alpha$ is thermal expansivity, $g_{0}$ is gravitational acceleration on the outer boundary at radius $r_{o}, q_{0}$ is the mean heat flux across the outer boundary, $D$ is shell thickness, $k$ is thermal conductivity, $\kappa$ is thermal diffusivity and $\nu$ is kinematic viscosity. The Ekman number represents the ratio of viscous and Coriolis forces

$$
E=\frac{\nu}{\Omega D^{2}}
$$


The Prandtl number is the ratio of kinematic viscosity to thermal diffusivity

$$
\operatorname{Pr}=\frac{\nu}{\kappa}
$$

and the magnetic Prandtl number is the ratio of kinematic viscosity to magnetic diffusivity $\lambda$

$$
P m=\frac{\nu}{\lambda}
$$

A fifth non-dimensional number is the amplitude of the outer boundary heat flux heterogeneity which is expressed by $q^{*}$ (see Eq. 2).

In all cases a volumetric homogeneous heat source $\epsilon$ compensates for the loss of heat through the outer boundary according to

$$
-4 \pi r_{o}^{2} \operatorname{Pr}\left[\frac{\partial T}{\partial r}\left(r_{o}\right)\right]=\frac{4}{3} \pi\left(r_{o}^{3}-r_{i}^{3}\right) \epsilon
$$

where $[. .$.$] denotes averaging over the outer boundary surface S$. In terms of the non-dimensional variables $\left[\frac{\partial T}{\partial r}\left(r_{o}\right)\right]=1$, so for $r_{i} / r_{o}=0.2$ (the geometry used in the study) the source term is $\epsilon \simeq 2.42$. We use moderate amplitudes of $\mathrm{CMB}$ heat flux heterogeneity to avoid violation of the Boussinesq approximation on which the dynamo code relies.

Most dynamo models fall into two categories. In the first, the radial field on the CMB is dominated by an axial dipole component, but the field does not reverse. In the second, the field is multipolar and dipole reversals occur (Kutzner and Christensen, 2002). Earth-like models that are both dipole-dominated and reversing are only found in a narrow transitional regime of parameters space (Olson, 2007; Wicht et al., 2009). Following Amit et al. (2011), we consider cases from both regimes.

\subsection{Hemispheric magnetic dichotomy monitoring}

The kinetic energy of the impactor is dissipated as a result of the irreversible work done by shock waves in damaging crustal rocks as well as heating and melting the target material (Pierazzo et al., 1997; Senshu et al., 2002; Reese and Solomatov, 2006; Monteux et al., 2011). After the excavation process, a significant fraction of the material molten by impact is redistributed heterogeneously at the surface of the impacted planet (Marinova et al., 2008, 2011). The distribution of the molten material is governed by the impact parameters such as the impact velocity 
and angle. At low impact velocities $(6-10 \mathrm{~km} / \mathrm{s})$ and oblique impact angles $\left(30-60^{\circ}\right)$, $50-70 \%$ of the impact-induced melt distribution might be contained within the area of impact and $25-30 \%$ might be deposited at the antipode of the impact site (Marinova et al., 2008). The impact-induced molten material might be redistributed over a thickness that ranges between 30 and $50 \mathrm{~km}$ (Marinova et al., 2008, 2011). The cooling and the crystallization of the molten material leads to the formation of the impact induced crust potentially recording the anomalous dynamo.

We consider two end member crust formation scenarios (Amit et al., 2011; Langlais and Thébault, 2011) as illustrated in Fig. 3. In the case of continuous and homogeneous crust formation, each part of the martian crust is formed by a large number of incremental and superposed additions (e.g. lava flows, sills, dykes) over an extended period of time. Each new layer records the magnetic field at its time of cooling below the Curie Temperature, and the present crustal field at a specific location results from the vertical superposition of the magnetization vectors of the various layers (Fig. 3, bottom line). If this crust formation scenario occurs while the dynamo reverses (Fig. 3, bottom right), the present local crustal field would thus represent the intensity of a long-term time-average martian paleomagnetic field. In the extreme case of periodic inversions and layers with equal thicknesses, the superposed opposite sign magnetization vectors could eventually cancel each other. The other end-member model assumes a random crust formation where crustal units are formed in relatively rapid events (Fig. 3, top line). Individual crustal blocks are created randomly both in space and time and each block acquires a magnetization which only depends on the dynamo field at the time of cooling. The present local field in this scenario thus results from adjacent magnetization vectors, or in a probabilistic way, from the time-average of the paleofield intensity. It is likely that neither one of these end-member scenarios represent what actually occurred on Mars, and rather that the actual way in which the magnetized part of the martian crust formed may be intermediate between the two scenarios.

We follow the statistical measures proposed by Amit et al. (2011) corresponding to these two end-member crust formation scenarios. In the context of a continuous homogeneous crust formation scenario, we calculate the ratio of intensities of the time-average field at the planet surface 


$$
S N_{c n t}=[|<\vec{B}>|]_{s h} /[|<\vec{B}>|]_{n h}
$$

where $S N$ denotes the ratio between the rms surface average in the southern and northern hemispheres and the subscript 'cnt' denotes continuous crust formation. In the context of a random crust formation scenario, we calculate the magnetic dichotomies based on the timeaverage of the magnetic field intensity

$$
S N_{r n d}=[<|\vec{B}|>]_{s h} /[<|\vec{B}|>]_{n h}
$$

where the subscript 'rnd' denotes random. Eqs. (9)-(10) are applied for the east-west dichotomies $E W_{c n t}$ and $E W_{r n d}$ by replacing the summations with the appropriate hemispheres.

The relevance of the continuous or random crust formation scenario depends on the relation between the cooling time of the Impact Induced Molten Material (IIMM) and the typical magnetic timescales. The cooling time of the IIMM strongly depends on the presence of an atmosphere that may prevent the IIMM from rapid cooling (e.g. Lebrun et al., 2013). In the case of molten material induced by a giant impact, a significant fraction of the atmosphere (if any) can be eroded from the impacted body (Shuvalov, 2009) which strongly enhances the excess heat removal and decreases the cooling and solidification times. Hence, the duration of the partially molten stage decreases from more than 1 million years with an atmosphere to $\sim 1000$ years when no atmosphere is present (Lebrun et al., 2013). The two crust formation scenarios give identical dichotomies for non-reversing dynamos (Amit et al., 2011). In the reversing case, if the crust was formed over a period much longer than a typical magnetic timescale, the continuous scenario is relevant (Dietrich and Wicht, 2013). Conversely, if the crust was formed faster than a typical magnetic timescale (i.e. without any atmosphere), the random scenario is relevant (see Fig. 3).

\section{Influence of impact heating on core dynamo}

Various CMB heat flux models corresponding to varying impactor sizes were examined. We considered two extreme geographic locations of the impactor, with a center falling on the geographical pole or on the equator. The impactor introduces warm material to the lower mantle, 
thus locally decreases the $\mathrm{CMB}$ heat flux. Apart from pattern dependence, the reduction in mean $\mathrm{CMB}$ heat flux (expressed by the $R a$ number compared to its reference homogeneous case) as well as the amplitude of the heat flux heterogeneity $q^{*}$ are all dependent in a selfconsistent manner on the radius of the impactor. A larger impactor warms a larger part of the $\mathrm{CMB}$, thus reduces the mean CMB heat flux $q_{0}$ more and produces larger $q^{*}$ (see Table 1 ).

Fig. 4 shows the time-average rms radial field at the $\mathrm{CMB}$ and the time-average field intensity at the surface of Mars for case 12 with an impactor of radius $800 \mathrm{~km}$ falling on the north geographic pole. Here and elsewhere all magnetic field values are given in units of $\sqrt{\rho \mu_{0} \lambda \Omega}$ where $\rho$ is the fluid density and $\mu_{0}$ is permeability of free space. The impactor yields an hemispheric field similar to those obtained with synthetic $Y_{1}^{0} \mathrm{CMB}$ heat flux patterns (Stanley et al., 2008; Amit et al., 2011; Dietrich and Wicht, 2013).

Fig. 5 shows the time-average zonal temperature and flow in case I2. The reduced CMB heat flux in the north pole region caused by the impactor results in a relatively warmer fluid there. The colder fluid in the more vigorously convecting southern hemisphere is associated with fluid downwelling at high-latitudes that concentrates the magnetic field. This produces a south-north magnetic hemispheric dichotomy. In addition, the boundary driven thermal wind flow exhibits a large one cell meridional circulation with surface flow going southward, carrying weak magnetic flux from north to south. These dynamical features are also in agreement with that obtained with a synthetic $Y_{1}^{0} \mathrm{CMB}$ heat flux pattern (Stanley et al., 2008; Amit et al., 2011).

When the impactor is falling on the equatorial plane, some magnetic dichotomy may be expected between eastern and western hemispheres. Indeed Fig. 6 shows that in case I5 such a dichotomy is obtained. Note that the east-west dichotomy in this case is significantly weaker than the south-north dichotomy in the corresponding polar impactor case I2 with the same impactor radius of $800 \mathrm{~km}$. However, compared to the east-west dichotomy obtained with a synthetic $Y_{1}^{1} \mathrm{CMB}$ heat flux pattern (Amit et al., 2011), the impactor driven east-west dichotomy is significantly stronger (compare cases I5 and Y3 in Table 1).

Fig. 7 shows two snapshots of the radial field at the CMB and the intensity at the surface of Mars for case I3 with the largest impactor studied here (radius of $1000 \mathrm{~km}$ ) falling on the 
north geographic pole as well as the corresponding long term time-average surface intensity. The differences between the two snapshots attest to the chaotic time dependence of this model. Nevertheless, in both snapshots the relatively small-scale radial field at the CMB is strongest at high latitudes of the southern hemisphere. The surface intensity is large scale, and also peaks at the polar region of the southern hemisphere. In this case, the time-average south-north dichotomy is 2.25 (see Table 1), very close to the lower bound estimation of 2.4 obtained by Amit et al. (2011) based on observations of the martian crustal magnetic field (Langlais et al., 2004).

Finally we examine the influence of the strength of the internal core convection. Case I4 is identical to case I 3 except for its $R a$ value which is larger. The south-north magnetic dichotomy in this case is 2.58 (Table 1), demonstrating that more vigorous core convection produces more hemispherical fields.

Our models show that a polar impactor leads to a stronger north-south hemispheric magnetic dichotomy than an equatorial impactor to an east-west dichotomy. We also find that in the non-reversing regime of parameters, the magnetic field dichotomy that can be recorded in the cooling IIMM is independent of the crust formation scenario (random or continuous). Reversing dynamos that convect stronger produce stronger magnetic dichotomies than nonreversing dynamos. These results are in agreement with those obtained by Amit et al. (2011) for degree-1 heat flux patterns.

The amplitude of the CMB heat flux heterogeneity (Fig. 2) is commonly measured by the peak to peak lateral variation (Olson and Christensen, 2002). However, when the pattern is not smooth and very localized, with imbalance between areas of positive and negative anomalies, $q^{*}$ is inadequate. This is exactly the case in the impact driven heat flux patterns considered here. For example, for very small impacts the amplitude is negligible but $q^{*}$ approaches 0.5 (Fig. 1b). We quantify the impact driven heterogeneity amplitude by $q_{0}^{r}$ (Eq. 1) which measures the extent of the CMB surface affected by the impact heating. Therefore, in order to compare the efficiency of magnetic hemispheric dichotomy generation by impact driven patterns with the efficiency by synthetic degree-1 patterns, $q_{0}^{r}$ in the first must be compared with $q^{*}$ in the latter. In addition we propose that the governing parameter controlling the amplitude of the 
hemispheric magnetic dichotomy is the horizontal Rayleigh number $R a_{h}$ (Willis et al., 2007).

We define $R a_{h}$ as:

$$
R a_{h}= \begin{cases}q^{*} R a & \text { in } \mathrm{Y} \text { cases } \\ q_{0}^{r} R a & \text { in I cases }\end{cases}
$$

To adequately compare the synthetic $Y_{1}^{0}$ CMB heat flux cases from Amit et al. (2011) and the polar impact driven CMB heat flux cases from this study, we plot in Fig. 8 the increase of the south-north dichotomy $S N-1$ as a function of $R a_{h}$ (11). This figure shows that for a given lateral forcing, an impact induced CMB heat flux heterogeneity is significantly more efficient than a synthetic $Y_{1}^{0} \mathrm{CMB}$ heat flux heterogeneity in generating hemispheric magnetic dichotomies. It also shows that the impactor size needed to generate the observed martian dichotomy (Amit et al., 2011) is slightly larger than $1000 \mathrm{~km}$, which is within the estimated range of impactor sizes (Marinova et al., 2008). The better efficiency of impact driven hemispheric dynamos over the synthetic cases is even more pronounced in the equatorial cases (see Table 1).

\section{Implication for crustal magnetic dichotomy}

Our results show that large $R a_{h}$ (and therefore large $R a$ ) are needed in order to get a dichotomy as strong as the observed. As large $R a$ are likely to be associated with reversing dynamos (e.g. Kutzner and Christensen, 2002), the answer depends on the duration of a chron relative to the duration of the crystallization of the IIMM induced by the impact. If a slow crystallization rate was coupled with short magnetic chrons (Dietrich and Wicht, 2013), then our models cannot explain the observed martian dichotomy. However, if a relatively rapid magma cooling was coupled with a relatively low reversal frequency then the impact induced magnetic dichotomy may have been recorded within the martian crust and explain the observed signal. Hence, the relevant magnetic timescale is a duration of a typical chron.

In our non-reversing dynamo models the chron duration is effectively infinite and the hemispheric magnetic dichotomies may be recorded by the crustal magnetization (Fig. 3, left). However, the amplitudes of the dichotomies in these cases are too low to explain the observed 
hemispheric crustal magnetic dichotomy on Mars (Table 1). Dynamo models with large amplitude hemispheric dichotomies tend to reverse frequently, in agreement with the findings of Dietrich and Wicht (2013). For example, in case I3 a typical chron persists for $\sim 18 \mathrm{kyr}$. In order for such a model to record an hemispheric magnetic dichotomy at the crust, very fast crust formation is required.

Dietrich et al. (2013) studied hemispherical dynamos in the framework of classical mean field theory. For $q^{*}>0.6$ Dietrich and Wicht (2013) found hemispheric $\alpha \Omega$ dynamos with fast oscillations over periods of $\sim 10$ kyrs. In our models the amplitude of CMB heat flux heterogeneity is moderate with $q^{*} \sim 0.6$, and more importantly $q_{0}^{r}<<0.6$. Our dynamos are therefore of the $\alpha^{2}$-type with chaotic (reversing or non-reversing) behaviour. In addition, the moderate $q_{0}^{r}$ does not change the dynamo regime from stable to reversing. In these dynamos the duration of a chron (or the reversal frequency) depends on the level of turbulence in the core (Olson and Amit, 2014), which is in general unknown (even for the Earth). Chron duration varied immensely over Earth's history between 40 kyrs - 40 Myrs (Merrill et al., 1998).

Fig. 9 illustrates the interplay between the relevant time-scales: the crystallization time $\tau_{c}$, the spreading time $\tau_{i m p}$ and the duration of a chron. In the context of a martian giant impact, the associated molten thickness $\delta$ ranges between 30 and $50 \mathrm{~km}$ and the melt fraction is $\sim$ $20 \%$ (Marinova et al., 2008). Hence, the crystallization and cooling times of the shallow IIMM induced by one impact should be rapid: even if the complete solidification timescale ultimately depends on the poorly constrained evolution of the post-impact transient atmosphere (Abe, 1997), crystallization of this local molten material should occur in less than $1000 \mathrm{yr}$ (Reese and Solomatov, 2006). The subsequent evolution of the partially molten material could involve isostatic readjustment of a deep, initially hemispheric, retained melt region and lateral spreading as a gravity current (Reese et al., 2011). Overall, the surface temperature of the IIMM falls below the Curie temperature in a timescale that strongly depends on factors such as the characteristics of the above atmosphere or the viscosity of the solid mantle if isostatic rebound is involved. This instant characterizes the beginning of the time interval when the solidified crust starts to record the magnetic field. The cold front then propagates downwards from the surface and a secondary front most probably develops at the base of the IIMM whose nature depends on the thermal state of the pre-impact crust on top of which the IIMM is 
superimposed (Fig. 9). The crustal material originating in the impact event stops recording the magnetic field precisely when the innermost region is cooler than the Curie temperature. In summary, given these somewhat overlapping ranges of timescales for the crust formation and magnetic chrons, we argue that both end-member crust formation scenarios are in principle possible, bearing in mind that reality may be somewhere in between.

Our study also emphasizes the importance of the duration of the post-impact thermal anomaly at the $\mathrm{CMB}\left(\tau_{i m p}\right)$. If this timescale is shorter than the time needed by the IIMM to cool down to temperatures smaller than the Curie temperature $\tau_{c}$, the hemispheric dynamo has ended before the crust was able to record it. However, if $\tau_{i m p}$ is larger than $\tau_{c}$, the postimpact magnetic field can be recorded within the crust. The beginning of this record starts at $\tau_{c, \text { top }} \sim 1 \mathrm{kyr}$ and ends at $\tau_{c, \text { int }} \sim 10-100 \mathrm{Myr}$, this time interval being mostly controlled by heat diffusion. The deep post-impact thermal re-adjustment occurs within a characteristic time that is governed by the rheology of the mantle surrounding the post-impact thermal anomaly. For a mantle viscosity comparable to that of the present day Earth, say around $10^{21} \mathrm{~Pa} . \mathrm{s}$, this characteristic spreading time is $\tau_{i m p} \sim 10-100$ Myr (Monteux et al., 2007; Watters et al., 2009) which is much larger than the characteristic magnetic diffusion time $\tau_{\lambda} \sim 30 \mathrm{kyrs}$ (e.g. Bloxham and Jackson, 1991) or the time needed by the top of the IIMM to reach the Curie temperature $\tau_{c, t o p}$ (Fig. 9).

\section{Conclusion}

Can the observed martian hemispheric magnetic dichotomy be the consequence of a giant impact that has led to an internal hemispheric magnetic field? Our results show that $\mathrm{a} \sim 1000$ $\mathrm{km}$ radius impactor can generate a sufficiently large scale thermal anomaly at the CMB so that a hemispheric dynamo is generated. An impact induced CMB heat flux heterogeneity is more efficient than a synthetic degree-1 CMB heat flux heterogeneity in generating strong hemispheric magnetic dichotomies. This magnetic dichotomy is stronger for a polar impact than for an equatorial impact. This result reconciles the giant impact induced scenario evoked to explain the martian topographic dichotomy (Andrews-Hanna et al., 2008; Marinova et al., 2008; Nimmo et al., 2008) and the heterogeneous CMB heat flux scenario proposed to explain 
why the magnetization is strong only in the southern hemisphere (Stanley et al., 2008) via a more realistic $\mathrm{CMB}$ heat flux pattern than the previously used synthetic degree-1. Hence, we propose here that an external event at the martian surface may have produced an internal hemispheric dynamo.

From our results, three relevant timescales arise: the cooling time of the impact induced molten material, the duration of a magnetic chron and the duration of the post-impact thermal anomaly at the CMB. The first is mainly governed by the characteristics of the martian atmosphere, the second is governed by the core dynamics while the third is governed by the mantle dynamics. If a relatively rapid magma cooling was coupled with a relatively low reversal frequency and with a stable CMB heat flux heterogeneity, then the impact induced magnetic dichotomy may have been recorded within the martian crust and explain the observed signal.

According to some studies (e.g. Arkani-Hamed and Olson, 2010), a giant impact might have led to a thermal stratification at the top of the core that terminated the dynamo. However, core stratification can be removed much faster by convection of molten material, over a timescale orders of magnitude shorter than the longevity of mantle thermal anomalies. Hence our scenario of giant impact leading to heterogenous mantle heating and to a hemispheric dynamo on Mars may follow an episode of dynamo shutdown.

Our impact driven CMB heat flux heterogeneity model may also be applied to model dynamos of other planets. Indeed, giant impacts were common in the later stage of accretion of terrestrial planets. The Earth is likely formed by accretion of a few dozen moon to Mars-size planetary embryos (see review by Chambers, 2004). A Mars-size impact on Earth may have resulted in the formation of the Moon (Hartmann and Davis, 1975; Cameron and Ward, 1976; Canup, 2004). The Moon was also probably hit by a large planetesimal at the end of its formation (Jutzi and Asphaug, 2011). Finally, an oblique collision of a large body with a mass about one sixth of Mercury's has likely stripped away a significant part of its mantle (Smith, 1979; Benz et al., 1988). Furthermore, these three terrestrial objects have or have had an internally generated magnetic field (Stevenson, 2003). Hence, giant impacts have potentially strongly influenced their internal dynamics and dynamo activities. 


\section{${ }_{499}$ Acknowledgments}

500 J. Monteux was funded by Agence Nationale de la Recherche (Accretis decision no. ANR501 10-PDOC-001-01). We thank Johannes Wicht for providing the MAGIC code. We thank 502 two anonymous reviewers for their careful and insightful reviews that greatly improve the 503 manuscript. The authors also thank N. Mangold and J.P. Lorand for useful discussions. 


\section{References}

Abe, Y., 1997. Thermal and chemical evolution of the terrestrial magma ocean. Phys. Earth Planet. Inter. 100, 27-39.

Acuña, M., Connerney, J., Wasilewski, P., Lin, R., Anderson, K., Carlson, C., McFadden, J., Curtis, D., Mitchell, D., Reme, H., Mazelle, C., Sauvaud, J., d’Uston, C., Cros, A., Medale, J., Bauer, S., Cloutier, P., Mayhew, M., Winterhalter, D., Ness, N., 1998. Magnetic field and plasma observations at Mars: Initial results of the Mars global surveyor mission. Science 279 (5357), 1676-1680.

Amit, H., Aubert, J., Hulot, G., 2010. Stationary, oscillating or drifting mantle-driven geomagnetic flux patches? J. Geophys. Res. 115, B07108.

Amit, H., Choblet, G., 2009. Mantle-driven geodynamo features - effects of post-Perovskite phase transition. Earth, Planets, and Space 61, 1255-1268.

Amit, H., Choblet, G., 2012. Mantle-driven geodynamo features - Effects of compositional and narrow D" anomalies. Phys. Earth Planet. Inter. 190, 34-43.

Amit, H., Christensen, U., Langlais, B., 2011. The influence of degree-1 mantle heterogeneity on the past dynamo of Mars. Phys. Earth Planet. Inter. 189, 63-79.

Anderson, B. J., Johnson, C. L., Korth, H., Winslow, R. M., Borovsky, J. E., Purucker, M. E., Slavin, J. A., Solomon, S. C., Zuber, M. T., McNutt, Jr., R. L., Dec. 2012. Low-degree structure in Mercury's planetary magnetic field. J. Geophys. Res. (Planets) 117, 0.

Andrews-Hanna, J. C., Zuber, M. T., Banerdt, W. B., 2008. The Borealis basin and the origin of the martian crustal dichotomy. Nature 453, 1212-1215.

Arkani-Hamed, J., Olson, P., 2010. Giant impact stratification of the Martian core. Geophys. Res. Lett. 37, L02201.

Asphaug, E., Agnor, C. B., Williams, Q., 2006. Hit-and-run planetary collisions. Nature 439, $155-160$. 
Aubert, J., Amit, H., Hulot, G., 2007. Detecting thermal boundary control in surface flows from numerical dynamos. Phys. Earth Planet. Inter. 160, 143-156.

Aubert, J., Amit, H., Hulot, G., Olson, P., 2008. Thermo-chemical wind flows couple Earth's inner core growth to mantle heterogeneity. Nature 454, 758-761.

Aubert, J., Labrosse, S., Poitou, C., 2009. Modelling the paleo-evolution of the geodynamo. Geophys. J. Int. 179, 1414-1428.

Benz, W., Slattery, W. L., Cameron, A. G. W., 1988. Collisional stripping of Mercury’s mantle. Icarus 74, 516-528.

Bloxham, J., 2002. Time-independent and time-dependent behaviour of high-latitude flux bundles at the core-mantle boundary. Geophys. Res. Lett. 29, 1854.

Bloxham, J., Jackson, A., 1991. Fluid flow near the surface of the Earth's outer core. Rev. Geophys. 29, 97-120.

Breuer, D., Labrosse, S., Spohn, T., 2010. Thermal Evolution and Magnetic Field Generation in Terrestrial Planets and Satellites. Space Sci. Rev. 152, 449-500.

Cameron, A. G. W., Ward, W. R., 1976. The Origin of the Moon. In: Lun. Planet. Inst. Conf. Abs. Vol. 7. pp. 120-122.

Canup, R. M., 2004. Simulations of a late lunar-forming impact. Icarus 168, 433-456.

Cao, H., Aurnou, J. M., Wicht, J., Dietrich, W., Soderlund, K. M., Russell, C. T., 2014. A dynamo explanation for Mercury's anomalous magnetic field. Geophys. Res. Lett. 41, 4127 4134.

Chambers, J. E., 2004. Planetary accretion in the inner Solar System. Earth Planet. Sci. Lett. $223,241-252$.

Chassefière, E., Langlais, B., Quesnel, Y., Leblanc, F., 2013. The fate of early Mars' lost water: The role of serpentinization. J. Geophys. Res. (Planets) 118, 1123-1134. 
Dietrich, W., Schmitt, D., Wicht, J., 2013. Hemispherical Parker waves driven by thermal shear in planetary dynamos. Europhys. Lett. 104.

Dietrich, W., Wicht, J., 2013. A hemispherical dynamo model: Implications for the Martian crustal magnetization. Phys. Earth Planet. Int. 217, 10-21.

Elkins-Tanton, L. T., Parmentier, E. M., Hess, P. C., 2003. Magma ocean fractional crystallization and cumulate overturn in terrestrial planets: Implications for Mars. Meteoritics and Planetary Science 38, 1753-1771.

Elkins-Tanton, L. T., Zaranek, S. E., Parmentier, E. M., Hess, P. C., 2005. Early magnetic field and magmatic activity on Mars from magma ocean cumulate overturn. Earth and Planetary Science Letters 236, 1-12.

Frey, H. V., Roark, J. H., Shockey, K. M., Frey, E. L., Sakimoto, S. E. H., 2002. Ancient lowlands on Mars. Geophys. Res. Lett. 29, 1384.

Gladman, B., Coffey, J., 2009. Mercurian impact ejecta: Meteorites and mantle. Meteoritics Planet. Sci. 44, 285-291.

Glatzmaier, G., Coe, R., Hongre, L., Roberts, P., 1999. The role of the earth's mantle in controlling the frequency of geomagnetic reversals. Nature 401, 885-890.

Golabek, G. J., Gerya, T. V., Kaus, B. J. P., Ziethe, R., Tackley, P. J., 2009. Rheological controls on the terrestrial core formation mechanism. Geochemistry, Geophysics, Geosystems 10, Q11007.

Gubbins, D., Willis, P. W., Sreenivasan, B., 2007. Correlation of Earth's magnetic field with lower mantle thermal and seismic structure. Phys. Earth Planet. Inter. 162, 256-260.

Harder, H., Christensen, U., 1996. A one-plume model of Martian mantle convection. Nature 380, 507-509.

Hartmann, W. K., Davis, D. R., 1975. Satellite-sized planetesimals and lunar origin. Icarus 24, $504-514$. 
Hori, K., Wicht, J., Christensen, U., 2010. The effect of thermal boundary conditions on dynamos driven by internal heating. Phys. Earth Planet. Inter. 182, 85-97.

Hori, K., Wicht, J., Dietrich, W., 2014. Ancient dynamos of terrestrial planets more sensitive to core-mantle boundary heat flows. Planet. Space. Sci. 98, 30-40.

Jutzi, M., Asphaug, E., 2011. Forming the lunar farside highlands by accretion of a companion moon. Nature 476, 69-72.

Ke, Y., Solomatov, V. S., 2006. Early transient superplumes and the origin of the Martian crustal dichotomy. Journal of Geophysical Research (Planets) 111, 10001.

Kutzner, C., Christensen, U., 2002. From stable dipolar towards reversing numerical dynamos. Phys. Earth Planet. Inter. 131, 29-45.

Kutzner, C., Christensen, U. R., 2004. Simulated geomagnetic reversals and preferred virtual geomagnetic pole paths. Geophys. J. Int. 157, 1105-1118.

Langlais, B., Amit, H., 2008. The past martian dynamo. Science 321, 1784-1785.

Langlais, B., Purucker, M., Mandea, M., 2004. Crustal magnetic field of Mars. J. Geophys. Res. 109, E02008.

Langlais, B., Thébault, E., 2011. Predicted and observed magnetic signatures of martian (de)magnetized impact craters. Icarus 212, 568-578.

Le Feuvre, M., Wieczorek, M. A., 2011. Nonuniform cratering of the Moon and a revised crater chronology of the inner Solar System. Icarus 214, 1-20.

Lebrun, T., Massol, H., Chassefière, E., Davaille, A., Marcq, E., Sarda, P., Leblanc, F., Brandeis, G., Jun. 2013. Thermal evolution of an early magma ocean in interaction with the atmosphere. Journal of Geophysical Research (Planets) 118, 1155-1176.

Lillis, R. J., Frey, H. V., Manga, M., 2008. Rapid decrease in Martian crustal magnetization in the Noachian era: Implications for the dynamo and climate of early Mars. Geophys. Res. Lett. 35, 14203. 
Lillis, R. J., Stewart, S. T., Manga, M., 2013. Demagnetization by basin-forming impacts on early Mars: Contributions from shock, heat, and excavation. J. Geophys. Res. (Planets) 118, $1045-1062$.

Marinova, M. M., Aharonson, O., Asphaug, E., 2008. Mega-impact formation of the Mars hemispheric dichotomy. Nature 453, 1216-1219.

Marinova, M. M., Aharonson, O., Asphaug, E., 2011. Geophysical consequences of planetaryscale impacts into a Mars-like planet. Icarus 211, 960-985.

Merrill, R., McElhinny, M., McFadden, P., 1998. The Magnetic Field of the Earth: Paleomagnetism, the Core, and the Deep Mantle. Academic Press, San Diego, California, USA.

Monteux, J., Arkani-Hamed, J., 2014. Consequences of giant impacts in early Mars: Core merging and Martian dynamo evolution. J. Geophys. Res. (Planets) 119, 480-505.

Monteux, J., Coltice, N., Dubuffet, F., Ricard, Y., 2007. Thermo-mechanical adjustment after impacts during planetary growth. Geophys. Res. Lett. 34, 24201-24205.

Monteux, J., Jellinek, A. M., Johnson, C. L., 2011. Why might planets and moons have early dynamos? Earth Planet. Sci. Lett. 310, 349-359.

Monteux, J., Jellinek, A. M., Johnson, C. L., 2013. Dynamics of core merging after a megaimpact with applications to Mars’ early dynamo. Icarus 226, 20-32.

Nimmo, F., Hart, S. D., Korycansky, D. G., Agnor, C. B., 2008. Implications of an impact origin for the martian hemispheric dichotomy. Nature 453, 1220-1223.

Olson, P., 2007. Gravitational dynamos and the low frequency geomagnetic secular variation. Proc. Nat. Acad. Sci. 104, 20159-20166.

Olson, P., Amit, H., 2014. Magnetic reversal frequency scaling in dynamos with thermochemical convection. Phys. Earth Planet. Inter. 229, 122-133.

Olson, P., Christensen, U., 2002. The time averaged magnetic field in numerical dynamos with nonuniform boundary heat flow. Geophys. J. Int. 151, 809-823. 
Olson, P., Coe, R. S., Driscoll, P. E., Glatzmaier, G. A., Roberts, P. H., 2010. Geodynamo reversal frequency and heterogeneous core-mantle boundary heat flow. Phys. Earth Planet. Inter. 180, 66-79.

Olson, P., Deguen, R., Hinnov, L. A., Zhong, S., 2013. Controls on geomagnetic reversals and core evolution by mantle convection in the Phanerozoic. Phys. Earth Planet. Inter. 214, 87-103.

Parker, R., 2003. Ideal bodies for Mars magnetics. JOURNAL OF GEOPHYSICAL RESEARCH-PLANETS 108 (E1).

Pierazzo, E., Melosh, H. J., 2000. Melt Production in Oblique Impacts. Icarus 145, 252-261.

Pierazzo, E., Vickery, A. M., Melosh, H. J., 1997. A Reevaluation of Impact Melt Production. Icarus $127,408-423$.

Pozzo, M., Davies, C., Gubbins, D., Alfè, D., 2012. Thermal and electrical conductivity of iron at Earth's core conditions. Nature 485, 355-358.

Quesnel, Y., Sotin, C., Langlais, B., Costin, S., Mandea, M., Gottschalk, M., Dyment, J., 2009. Serpentinization of the martian crust during Noachian. Earth Planet. Sci. Lett. 277, 184-193.

Reese, C. C., Orth, C. P., Solomatov, V. S., 2010. Impact origin for the Martian crustal dichotomy: Half emptied or half filled? J. Geophys. Res. (Planets) 115 (14), 5004-+.

Reese, C. C., Orth, C. P., Solomatov, V. S., 2011. Impact megadomes and the origin of the martian crustal dichotomy. Icarus 213, 433-442.

Reese, C. C., Solomatov, V. S., 2006. Fluid dynamics of local martian magma oceans. Icarus $184,102-120$.

Reese, C. C., Solomatov, V. S., 2010. Early martian dynamo generation due to giant impacts. Icarus 207, 82-97.

Roberts, J., Zhong, S., 2006. Degree-1 convection in the Martian mantle and the origin of the hemispheric dichotomy. J. Geophys. Res. 111, E06013. 
Roberts, J. H., Arkani-Hamed, J., 2012. Impact-induced mantle dynamics on Mars. Icarus 218, 278-289.

Roberts, J. H., Lillis, R. J., Manga, M., 2009. Giant impacts on early Mars and the cessation of the Martian dynamo. J. Geophys. Res. (Planets) 114 (13), E04009.

Rochette, P., 2006. Crustal magnetization of Mars controlled by lithology or cooling rate in a reversing dynamo? Geophys. Res. Lett. 33, 2202.

Schubert, G., Spohn, T., 1990. Thermal history of Mars and the sulfur content of its core. J. Geophys. Res. 95, 14095-14104.

Senshu, H., Kuramoto, K., Matsui, T., 2002. Thermal evolution of a growing Mars. J. Geophys. Res. 107, 1-13.

Shuvalov, V., Oct. 2009. Atmospheric erosion induced by oblique impacts. Meteoritics and Planetary Science 44, 1095-1105.

Smith, J. V., 1979. Mineralogy of the Planets: a Voyage in Space and Time. Mineralogical Magazine 43, 1-89.

Solomon, S. C., Aharonson, O., Aurnou, J. M., Banerdt, W. B., Carr, M. H., Dombard, A. J., Frey, H. V., Golombek, M. P., Hauck, S. A., Head, J. W., Jakosky, B. M., Johnson, C. L., McGovern, P. J., Neumann, G. A., Phillips, R. J., Smith, D. E., Zuber, M. T., 2005. New Perspectives on Ancient Mars. Science 307, 1214-1220.

Stanley, S., 2010. A dynamo model for axisymmetrizing Saturn's magnetic field. Geophys. Res. Lett. 37, 5201.

Stanley, S., Elkins-Tanton, L., Zuber, M. T., Parmentier, E. M., 2008. Mars’ Paleomagnetic Field as the Result of a Single-Hemisphere Dynamo. Science 321, 1822-1825.

Stevenson, D. J., 2003. Planetary magnetic fields. Earth Planet. Sci. Lett. 208, 1-2.

Tonks, W. B., Melosh, H. J., 1992. Core formation by giant impacts. Icarus 100, 326-346. 
Šrámek, O., Ricard, Y., Dubuffet, F., 2010. A multiphase model of core formation. Geophys. J. Int. 181, 198-220.

Watters, W. A., Zuber, M. T., Hager, B. H., 2009. Thermal perturbations caused by large impacts and consequences for mantle convection. J. Geophys. Res. (Planets) 114, E02001.

Wicht, J., 2002. Inner-core conductivity in numerical dynamo simulations. Phys. Earth Planet. Inter. 132, 281-302.

Wicht, J., Stellmach, S., Harder, H., 2009. Numerical models of the geodynamo: From fundamental Cartesian models to 3D simulations of field reversals. In: Glassmeier, H., Soffel, H., Negendank, J. (Eds.), Geomagnetic Field Variations - Space-time structure, processes, and effects on system Earth. Springer, Berlin, pp. 107-158.

Willis, A. P., Sreenivasan, B., Gubbins, D., 2007. Thermal core mantle interaction: Exploring regimes for "locked" dynamo action. Phys. Earth Planet. Inter. 165, 83-92.

Yoder, C. F., Konopliv, A. S., Yuan, D. N., Standish, E. M., Folkner, W. M., 2003. Fluid Core Size of Mars from Detection of the Solar Tide. Science 300, 299-303.

Zhong, S., Zuber, M. T., 2001. Degree-1 mantle convection and the crustal dichotomy on Mars. Earth and Planetary Science Letters 189, 75-84.

Zuber, M. T., 2001. The crust and mantle of Mars. Nature 412, 220-227. 


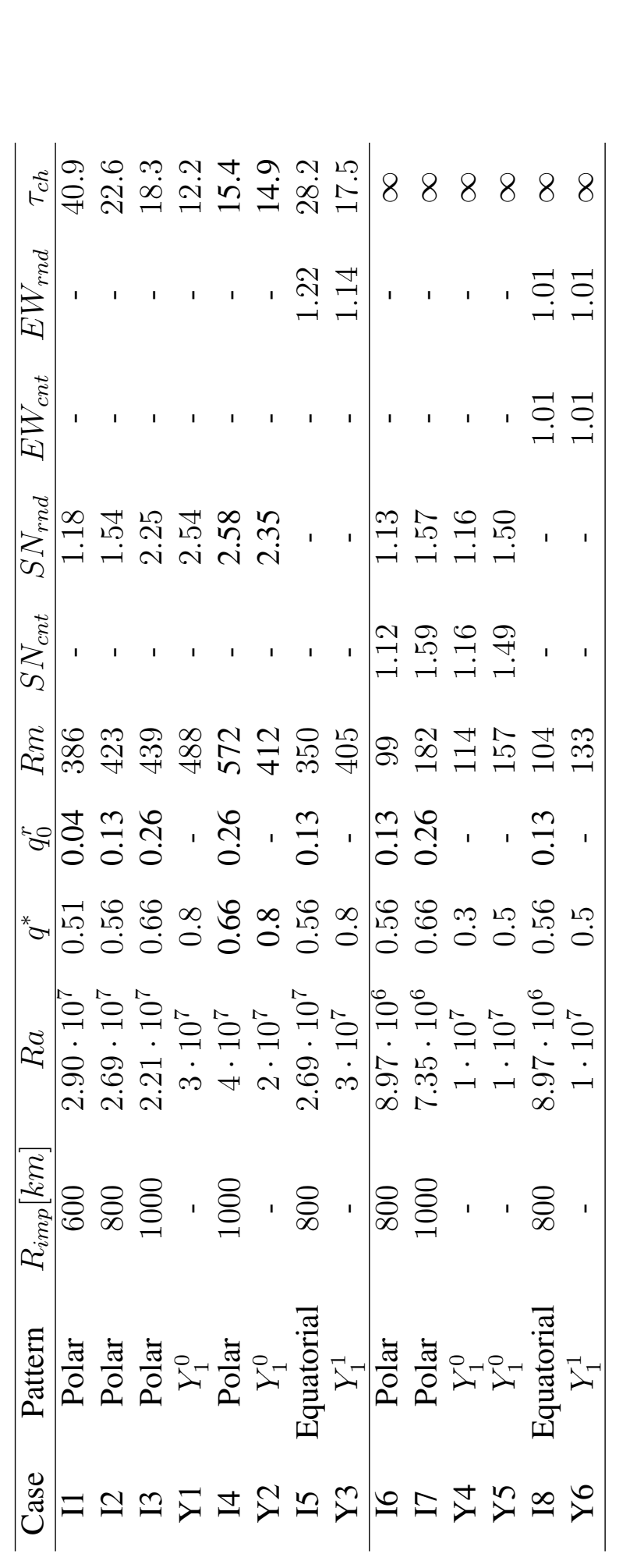

॥

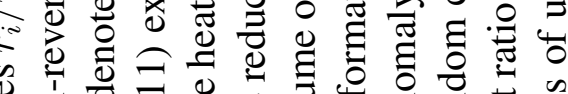

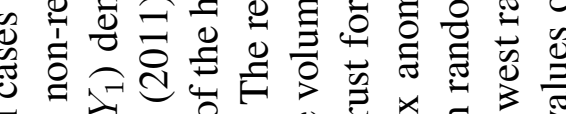
ส

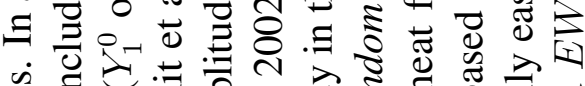

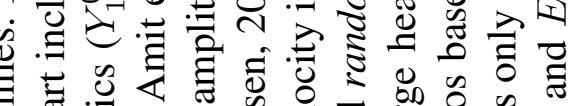

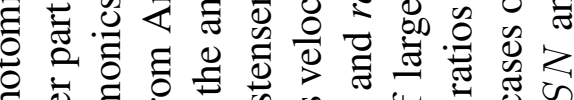
पे

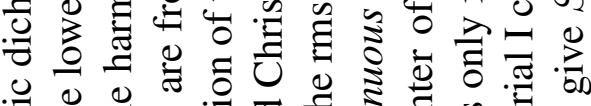

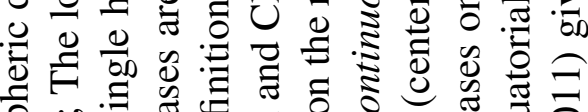

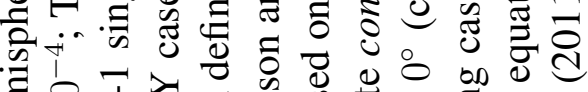

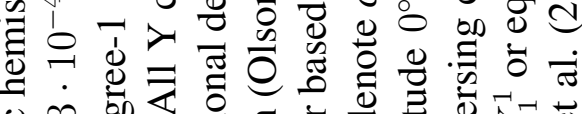

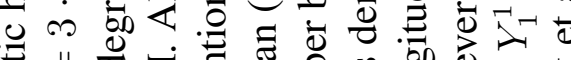

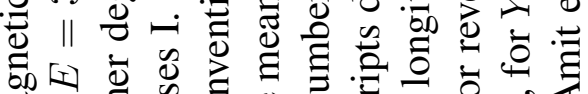

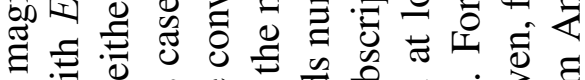

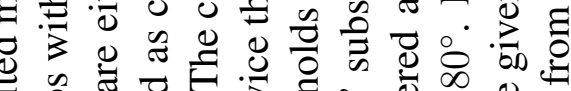

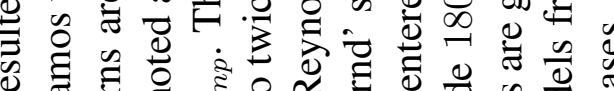

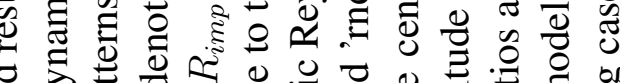

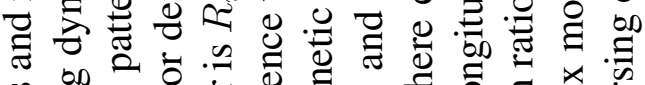

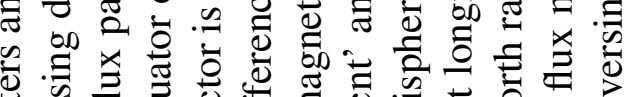

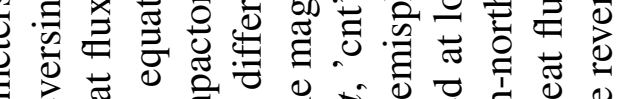

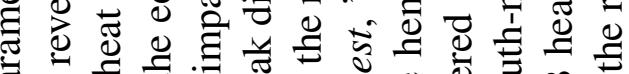

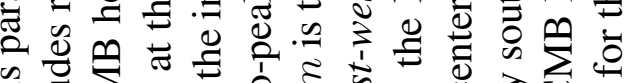

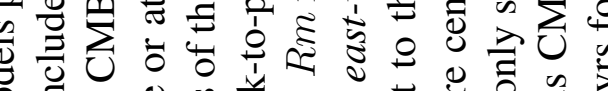

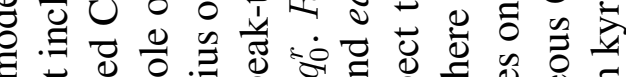

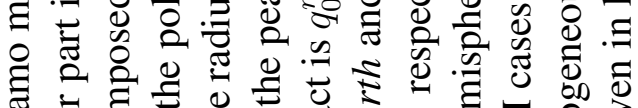
పี

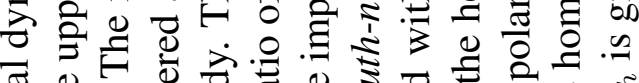
ฮี

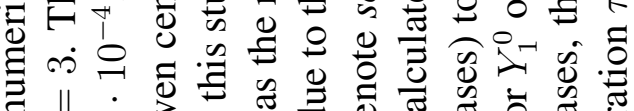

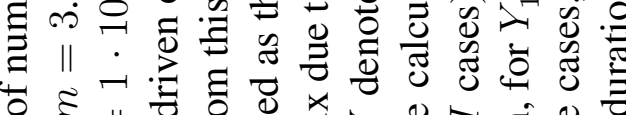

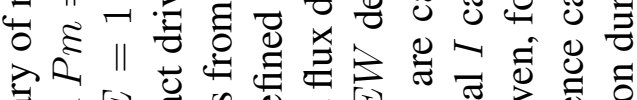

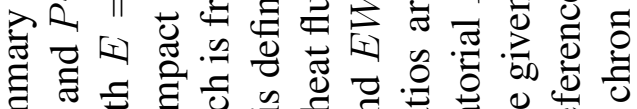

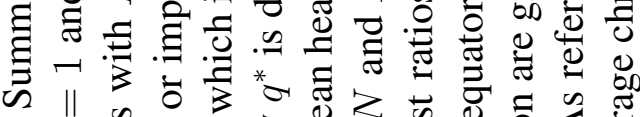

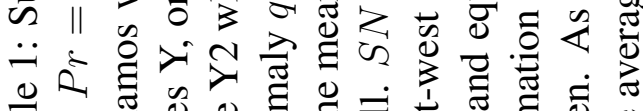

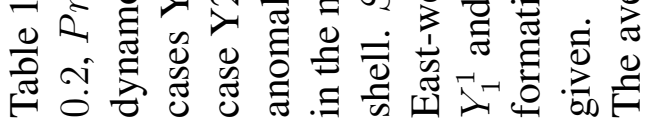


a)

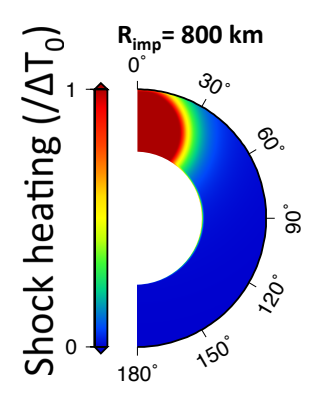

b)

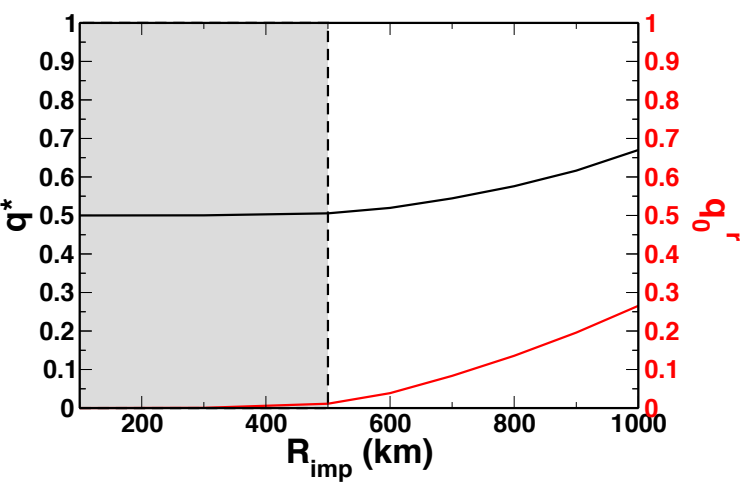

Figure 1: a) martian mantle post-impact temperature increase for $R_{i m p}=800 \mathrm{~km}$. b) Corresponding conventional amplitude of heat flux heterogeneity on the CMB $q^{*}$ (black line, Eq. 2 ) and the mean heat flux reduction $q_{0}^{r}$ (red line, Eq. 1) vs. impactor size $R_{i m p}$. In the grey domain $\left(R_{i m p}<500 \mathrm{~km}\right)$, the impactor is too small for isobaric core to reach the CMB.

a)

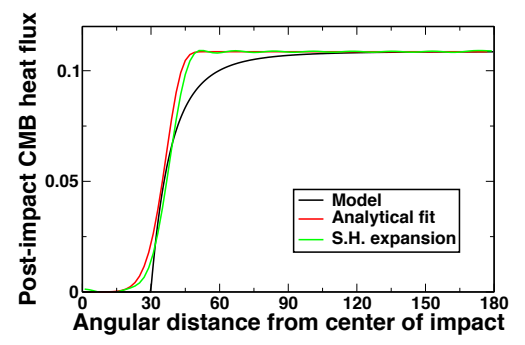

b)
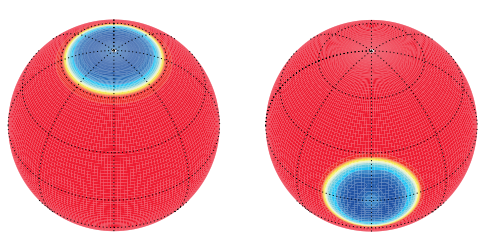

Figure 2: a) Post-impact CMB heat flux (black line), analytical fit (red line) and spherical harmonic expansion (green line) vs. angular distance from the center of the impact driven heated area. b) Imposed CMB heat flux anomalies resulting from an impactor of radius 800 $\mathrm{km}$ falling on the north pole (left) or the equator (right). 


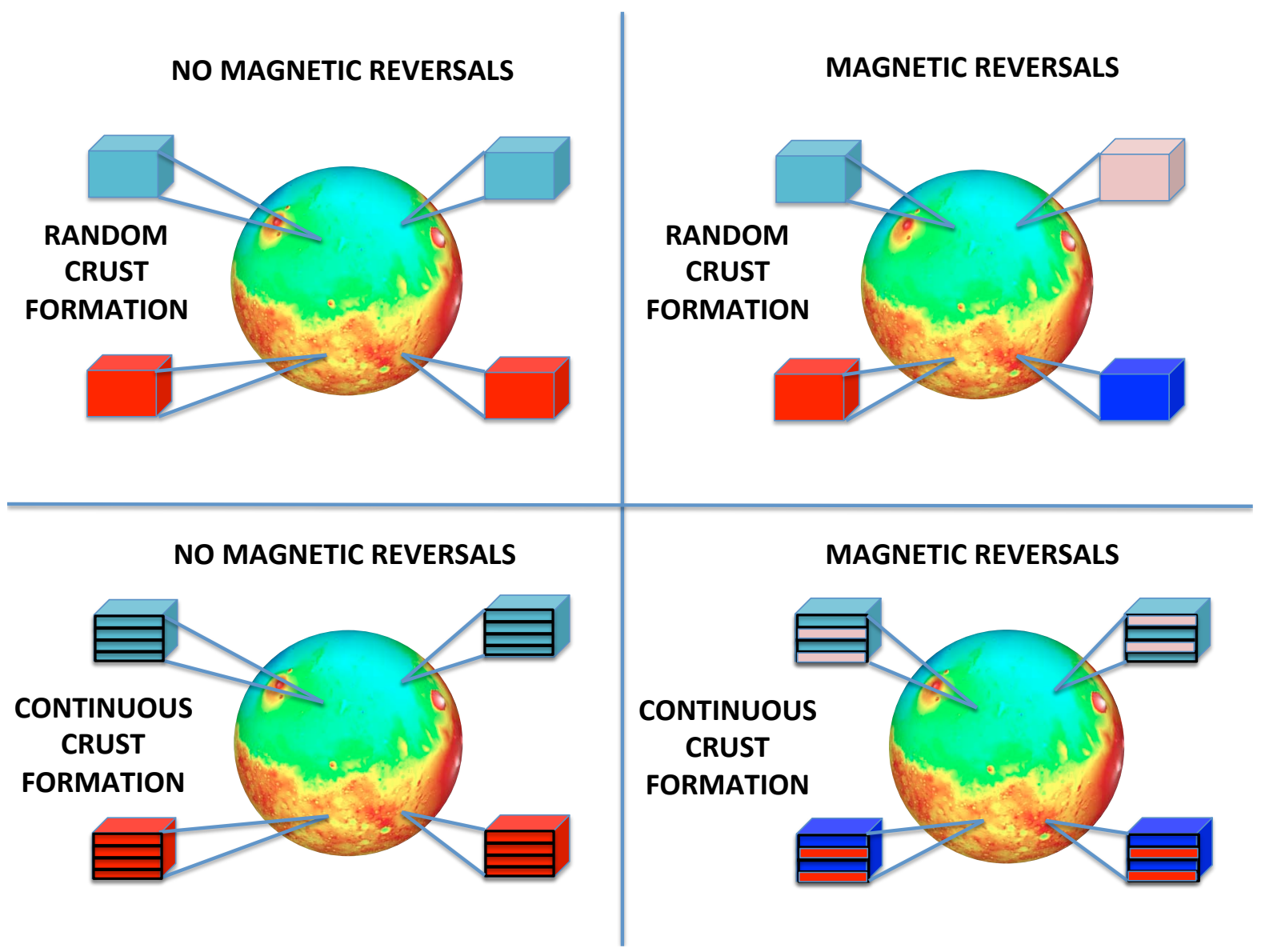

Figure 3: Schematic illustration of the random (top) and continuous (bottom) crust formation scenarios and the corresponding recorded magnetization without (left) or with (right) magnetic reversals. Blue/red denotes negative/positive radial magnetic paleofields respectively, so alternating colors correspond to paleomagnetic reversals. Cubes represent a vertical cut through the martian crust that has recorded strong (deep blue or deep red) or weak (light red or light blue) magnetic paleofields. The weak magnetic paleofield is restricted to the impacted pole while the strong magnetic paleofield is restricted to the opposite one. The current magnetic field observed at the surface results from the vertically integrated magnetization over the cube. 

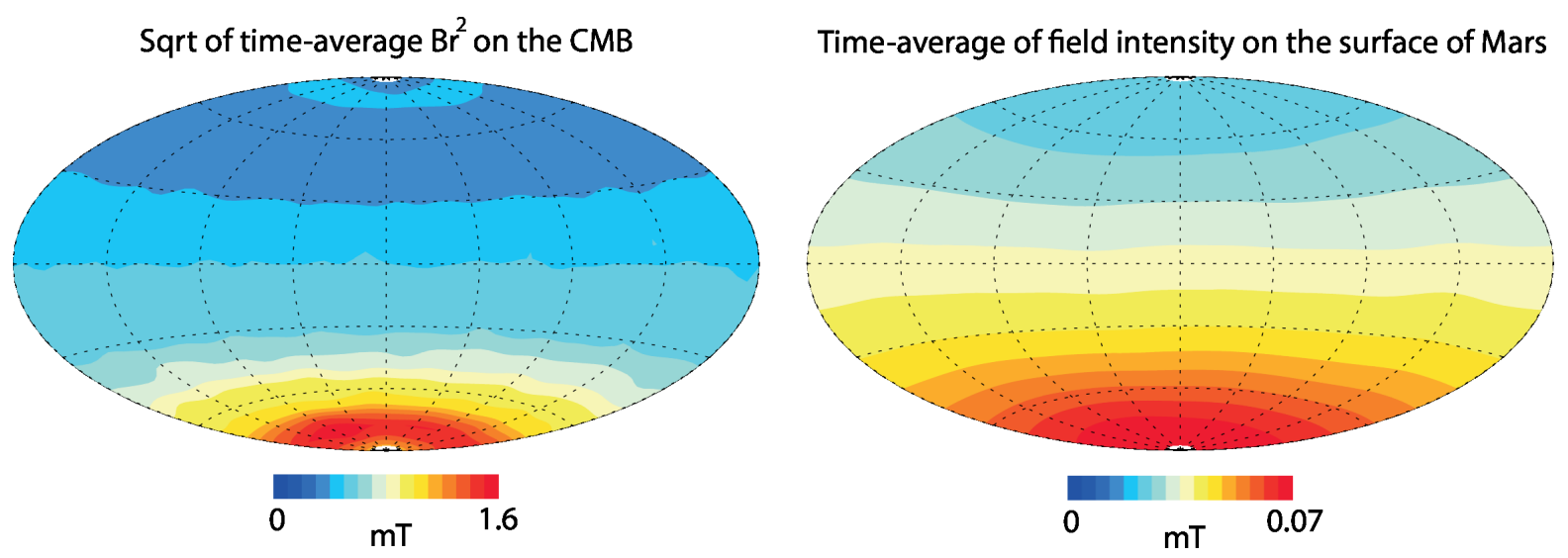

Figure 4: Time-average magnetic field properties on the CMB (left) and on the surface of Mars (right) in case I2. The CMB field is upward continued to the surface of Mars as a potential field.
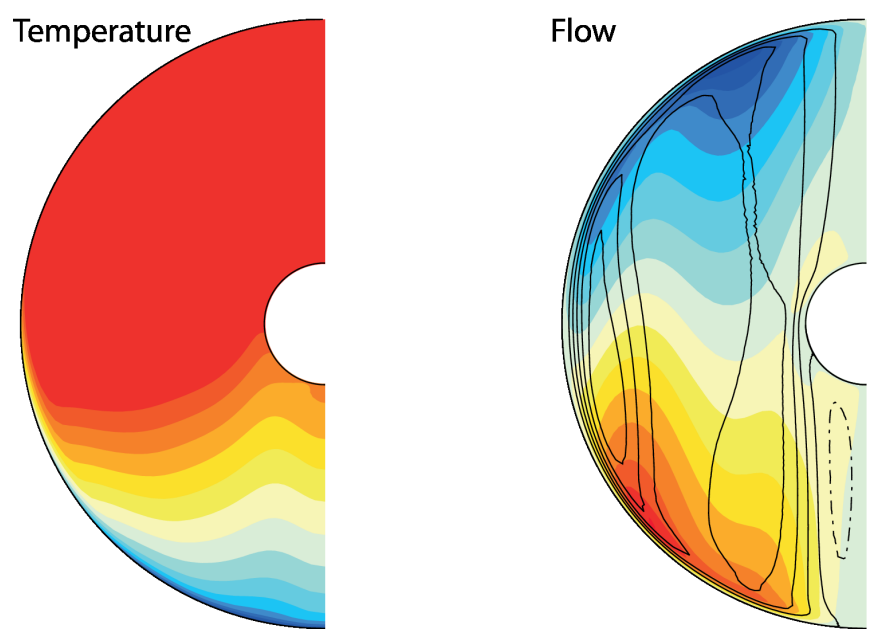

Figure 5: Time-average zonal temperature (left) and flow (right) in case I2. In the right subplot colors denote azimuthal flow and streamlines denote meridional circulation (solid/dashed are anti-clockwise/clockwise respectively). 

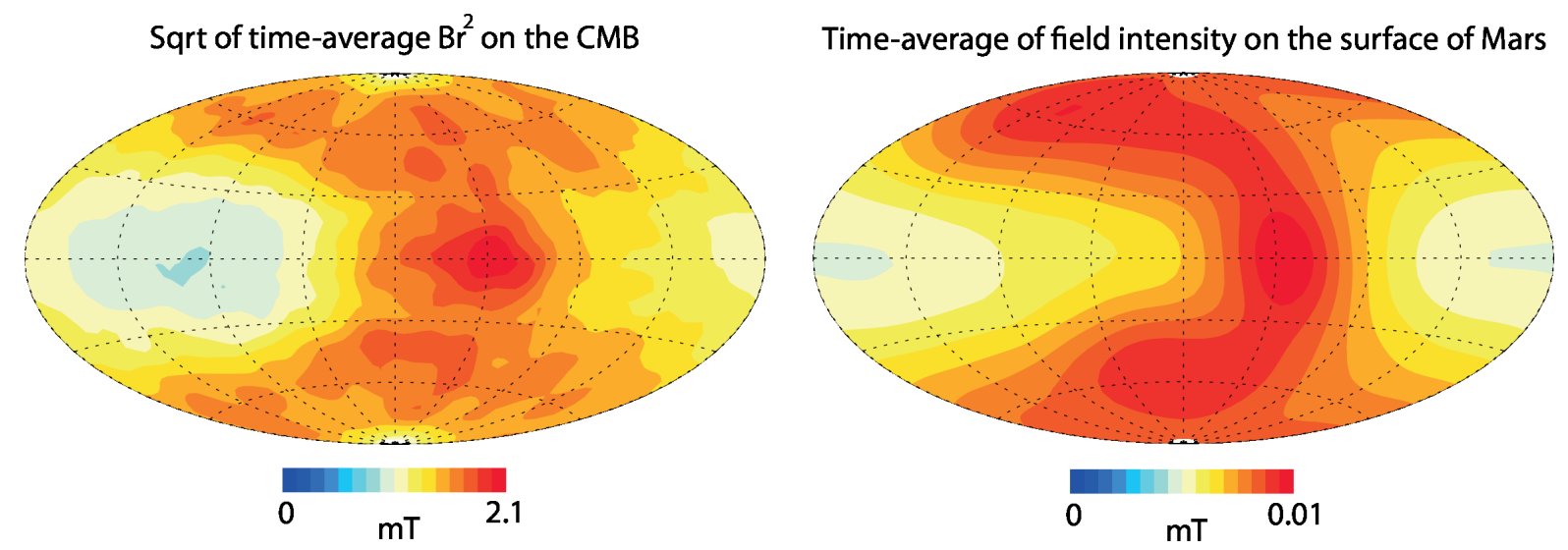

Figure 6: As in Fig. 4 for case I5 (note different color scales). 


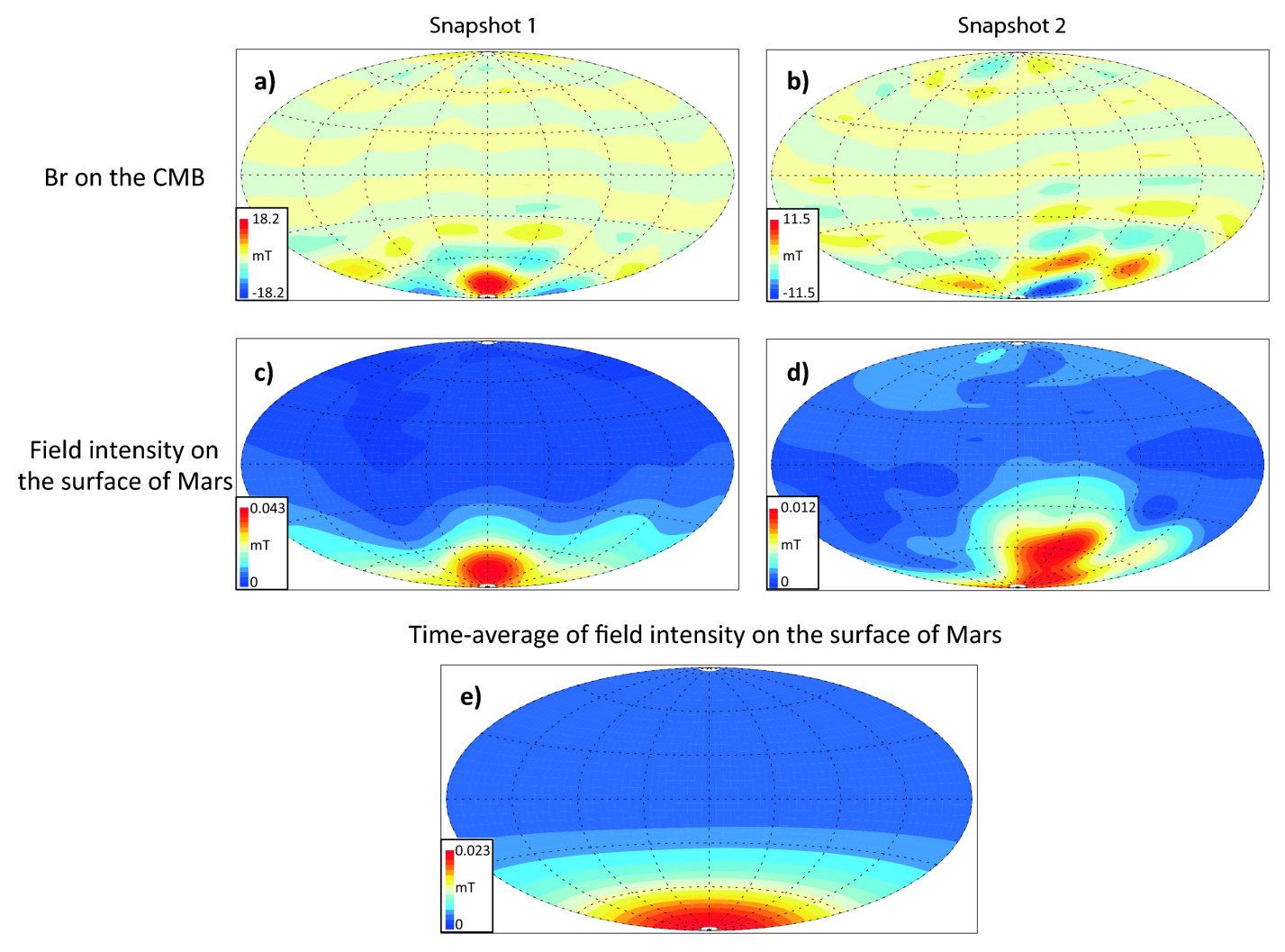

Figure 7: Two arbitrary snapshots (two first lines) of the radial magnetic field $B_{r}$ on the coremantle boundary CMB ( $a$ and $b$ ) and surface intensity of the magnetic field (c and d) in case I3. The time-average surface intensity is shown on the third line (e). Here the spherical harmonic expansion is truncated at $\ell_{\max }=10$. Note differences in color scales among the maps. The CMB field is upward continued to the surface of Mars as a potential field. 


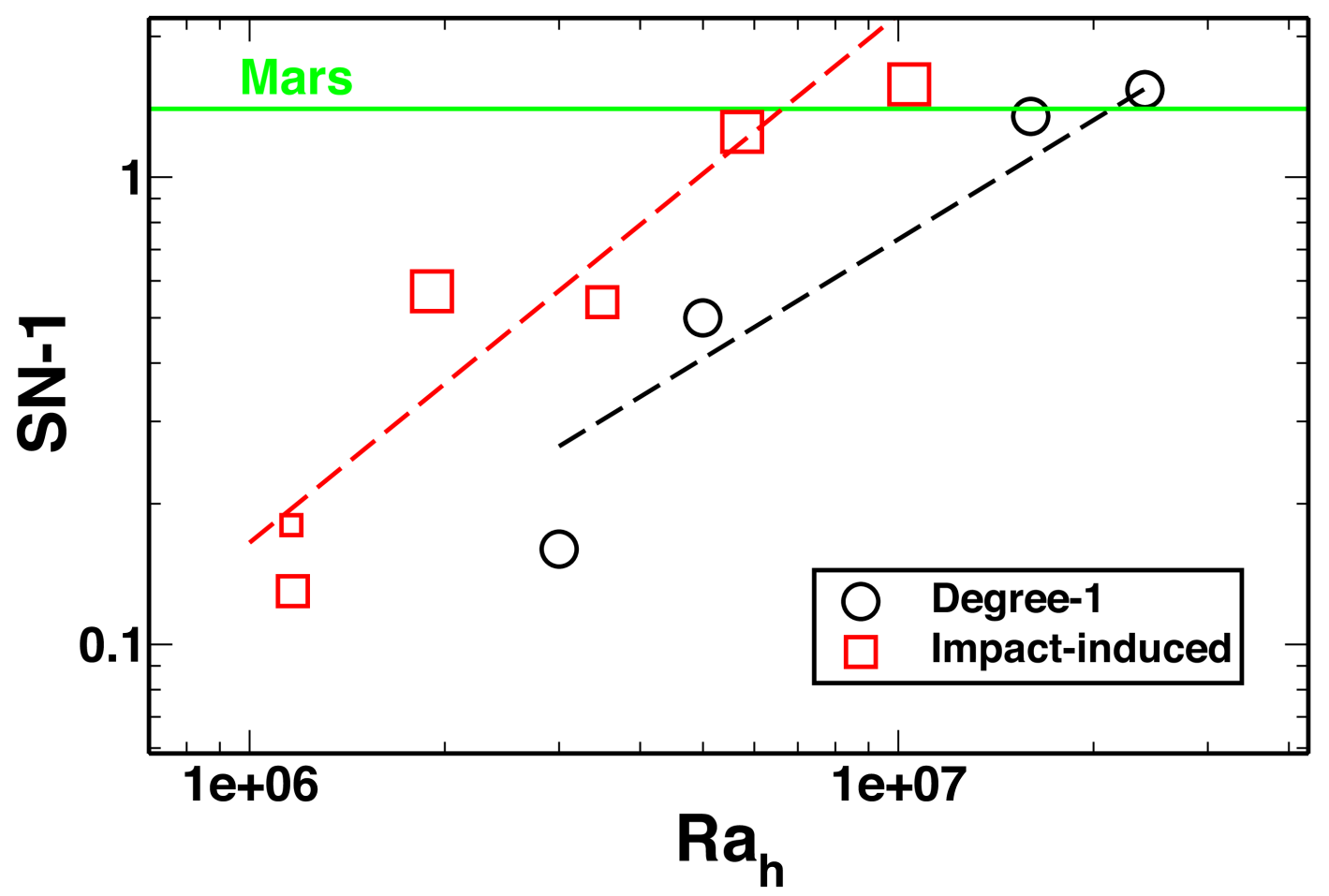

Figure 8: South-north dichotomy as a function of the horizontal Rayleigh number $R a_{h}$ (Eq. 11). The black symbols are the values obtained (with $r_{i} / r_{o}=0.2$ ) for the synthetic $Y_{1}^{0} \mathrm{CMB}$ heat flux patterns (with $R a_{h}=q^{*} R a$ ). The red symbols are the values obtained here in cases of polar impact driven CMB heat flux patterns (with $R a_{h}=q_{0}^{r} R a$ ). The size of the red symbols increases with the size of the impactor. The corresponding power law fits are plotted with dashed lines. The green horizontal line represents the martian value of $S N-1$ from Amit et al. (2011) based on observations of the martian crustal magnetic field (Langlais et al., 2004). All degree-1 cases are from Amit et al. (2011) except case Y2 from this study (see also Tab. $1)$. 


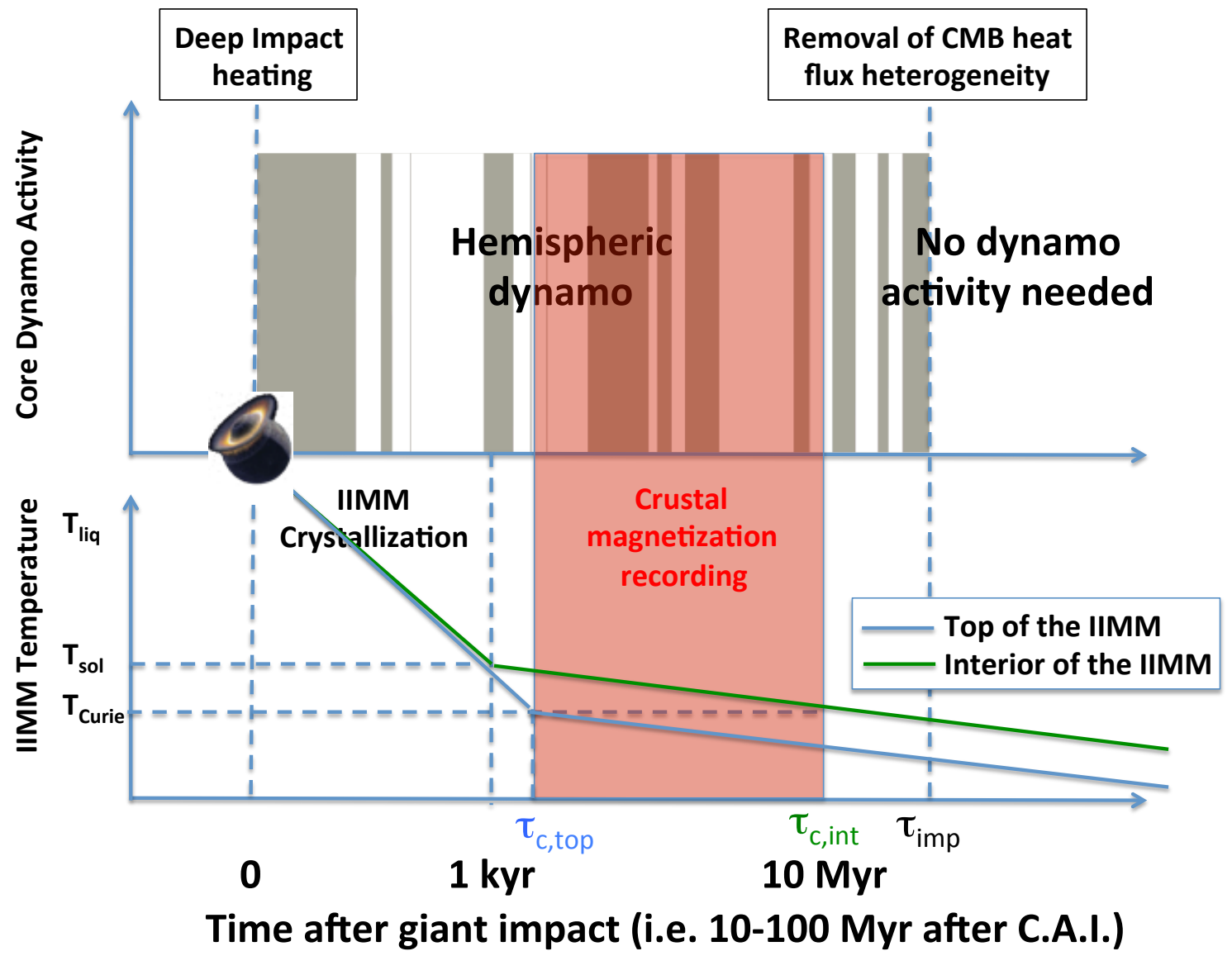

Figure 9: Schematic illustration of temporal evolution of core dynamo activity (top panel) and of the IIMM temperature (bottom panel) after a giant impact. The C.A.I. (Calcium-Aluminium rich Inclusions) are the oldest objects in the solar system. In the bottom panel, the blue line represents the temperature evolution at the interior of the IIMM while the green line represents the top of the IIMM (i.e. the post impact martian surface). 